04

\title{
Об интерферометрическом способе измерения коэффициентов отражения
}

\author{
(C) А.А. Ковалёв \\ Институт ффизики полупроводников им. А.В. Ржанова Сибирского отделения РАН, \\ 630090 Новосибирск, Россия \\ e-mail: kovalev@isp.nsc.ru
}

Поступила в редакцию 22.12.2017 г.

Предложен способ определения коэффициента отражения для оптических элементов, основанный на

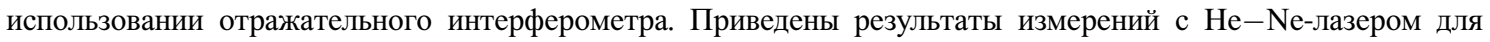
нескольких образцов с различным уровнем отражательной способности. Проведено сравнение с результатами измерений обычным способом. Оценен выигрыш от использования отражательного интерферометра.

DOI: $10.21883 /$ OS.2018.06.46086.311-17

\section{Введение}

Наиболее простой способ определить коэффициент отражения образца - это измерить сигнал фотоприемника от падающего на образец пучка излучения малой интенсивности $I_{1}$, а затем измерить сигнал от отраженного образцом пучка $I_{2}$, не допуская при этом включения в оптическую схему дополнительных элементов, которые могут изменять параметры пучков. Коэффициент отражения $R$ при этом будет равен $R=I_{2} / I_{1}$. В этом способе имеются достаточно большие трудности при измерениях вблизи $R=1$, когда определение коэффициента отражения проводится по измерениям двух больших сигналов, близких по величине. Это вынуждает прибегать к компенсационным схемам (электрическим или оптическим), не свободным от различных флуктуаций сигналов.

В интерферометрии сигналы пропускания и отражения характеризуются амплитудами коэффициентов отражения внутрь интерферометра, $r=R^{1 / 2}[1]$. Свойства интерферометров в отражении хорошо исследованы и используются для селекции мод в лазерах $[2,3]$ и в фазовых измерениях [4].

В интерферометрии в измеряемых сигналах участвует только когерентная часть поля отраженной волны. Рассеянная часть, которая при обычном способе измерений может попадать на чувствительный элемент фотодетектора, в интерференционный сигнал входит в меньшей степени.

Имеется принципиальная возможность наблюдать интерференционную картину в отраженном свете в расходящемся пучке, но для этого нужен широкий пучок, что уже является недостатком, так как требует одинаковости свойств образца на всем поперечнике пучка. Сигналы измеряются в точках минимума $S_{\min }$ и максимума $S_{\max }$ интерференционной картины. Контраст интерференции определяется как $\Delta S / S_{\max }=\left(S_{\max }-S_{\min }\right) / S_{\max }$. Следует отметить, что дифракция приводит к некоторому размыванию картины и, следовательно, к существенным ошибкам, т. е. $\Delta S / S \neq \Delta R / R$.

\section{Измерения коэффициента отражения с помощью сканируемого отражательного интерферометра}

Другой вариант наблюдения интерференции в отраженном свете заключается в сканировании базы интерферометра. Схема измерений с использованием $\mathrm{He}-\mathrm{Ne}$-лазера 1 показана на рис. 1. В качестве интерферометрического устройства используется интерферометр Фабри-Перо 4, работающий на отражение (RI Reflective Interferometer). На вставке слева на рис. 1 он показан с обозначениями базы $l(U)$, сканируемой при подаче переменного напряжения $U$ на пьезоэлемент (место ввода напряжения обозначено цифрой 5 на рис. 1), а также с коэффициентами отражения переднего зеркала $r_{1}$, образца $r_{2}$ и интерферометра $r$. В качестве переднего зеркала используется пластина из оптически прозрачного в рассматриваемой области спектра материала, имеющая клин, который предотвращает попадание отраженного от внешней грани пластины излучения в фотодетектор. Переднее зеркало RI закреплено на пьезотрансляторе. Вторым зеркалом RI является исследуемый образец.

Амплитуда коэффициента отражения пластины при углах падения излучения, близких к нормали, составляет $r_{1}=(n-1) /(n+1)$, где $n-$ показатель преломления материала пластины на рабочей длине волны. Коэффициент отражения образца по электрическому полю излучения равен $r_{2} \exp [i(\delta+\Phi)]$. Разность хода волн в RI равна $\delta=(4 / \lambda) l \cos \theta$, где $\theta-$ угол наклона пучка к нормали в интерферометре; в наших экспериментах $\cos \theta \approx 1$. Коэффициент отражения для падающей плоской волны выражается формулой

$|r|^{2}=\left|r_{1}+\left(\tau r_{2} \exp [i(\delta+\Phi)]\right) /\left(1+r_{1} r_{2} \exp [i(\delta+\Phi)]\right)\right|^{2}$,

где $\tau=1-r_{1}^{2}$ при отсутствии потерь на переднем зеркале, $i$ - мнимая единица. На вставке в нижней части рис. 1 показаны зависимости коэффициента отражения от полной фазы $(\delta+\Phi)$, где $\delta \propto l \propto U$. Здесь может 

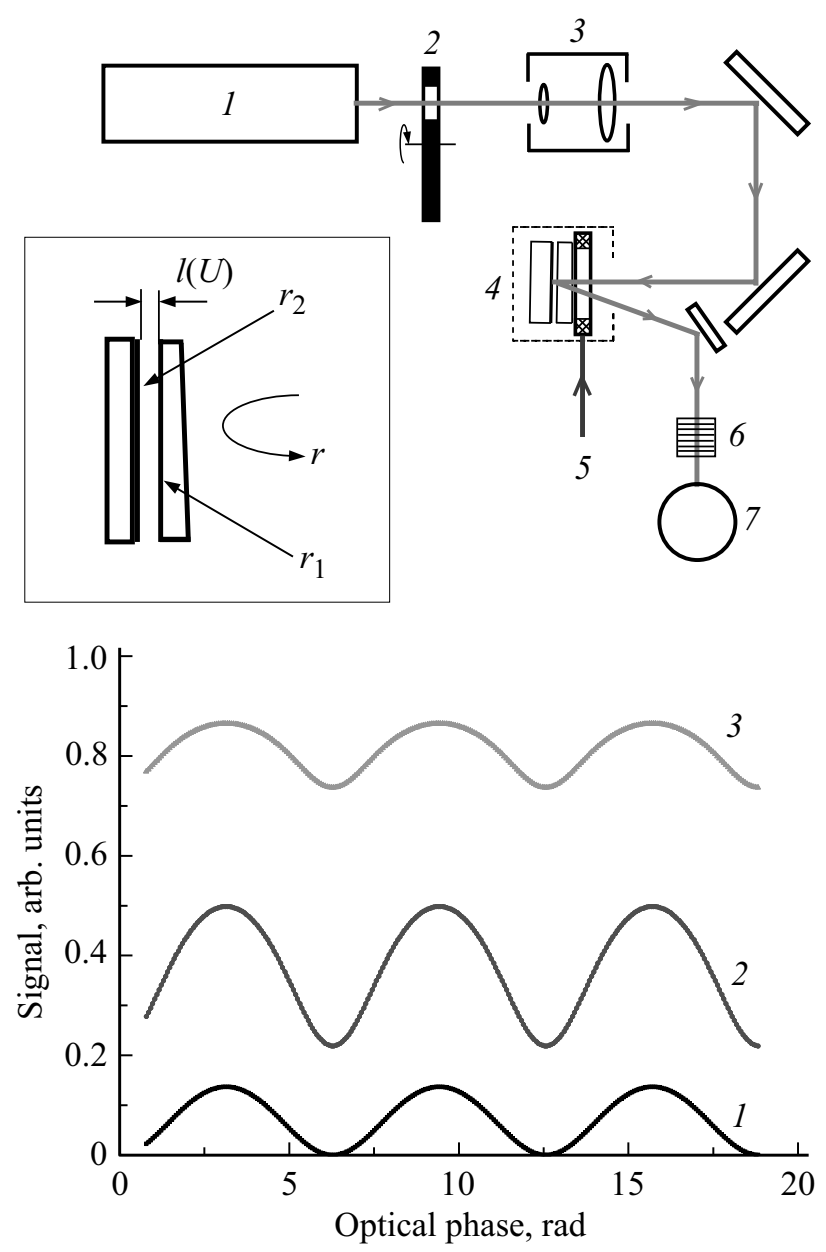

Рис. 1. Оптическая схема измерения коэффициента отражения с помощью отражательного интерферометра: 1 - источник излучения - He-Ne-лазер, 2 - прерыватель пучка излучения, 3 - коллиматор, 4 - отражательный интерферометр (RI - Reflective Interferometer), 5 - ввод переменного напряжения $U$, подаваемого на пьезоэлемент для сканирования базы RI, 6 - ослабители, 7 - фотодетектор. На вставке слева в средней части рисунка показан отражательный интерферометр со сканируемой базой $l(U)$ и коэффициентами отражения переднего зеркала $r_{1}$, образца $r_{2}$ и интерферометра $r$. Вставка внизу показывает вид сигнала для трех значений коэффициента отражения переднего зеркала RI: $1-r_{2}=0.2,2-0.6$, $3-0.9$.

быть реализован небольшой поперечный размер пучка, и появляется возможность исключить влияние дифракции на результат.

Измерения проводились со стабильным по амплитуде гелий-неоновым лазером (обозначен цифрой 1 на рис. 1) $(\lambda=0.6328$ мкм) мощности не более 1 мВт. В спектре излучения лазера отсутствовали другие линии. Прерыватель пучка 2 использовался для определения $S_{\max }$. Коллиматор 3 позволил расположить перетяжку гауссова пучка на поверхности интерферометра при диаметре $\leq 300$ мкм. Угол между падающим и отраженным пучками составлял $\approx 3^{\circ}$. Калиброванный ослабитель 6 служил для измерений $S_{\max }$ в линейном режиме фотодетектора. Изменение базы RI в пределах $\leq 300$ мкм не оказывало заметного влияния на результаты измерений. Зависимость величины

$$
\left(R_{\max }-R_{\min }\right) / R_{\max }=\Delta R / R_{\max }=\Delta S / S_{\max }
$$

от амплитуды коэффициента отражения $r_{2}$ приведена на рис. 2, $a$.

В качестве материалов для переднего зеркала интерферометра рассмотрены кварц $(n=1.45)$, лейкосапфир $(n=1.76)$ и селенид цинка $(n=2.89)$. Легко заметить, что наименьшая точность измерений получается в области $r_{1} \approx r_{2}$; кроме того, здесь отсутствует однозначное определение $r_{2}$, так как одному и тому же $\Delta S / S_{\max }$ соответствует два различных значения $r_{2}$. Это приводит к необходимости некоторых априорных знаний об области $r_{2}$, что может быть получено из величины $S_{\max }$. Для устранения неточностей, исходя из априорных значений $r_{2}$, нужно выбирать материал переднего зеркала: если мы попадаем в область максимума для кварцевого переднего зеркала, то для зеркал из сапфира или селенида цинка будет сохранена высокая чувствительность.
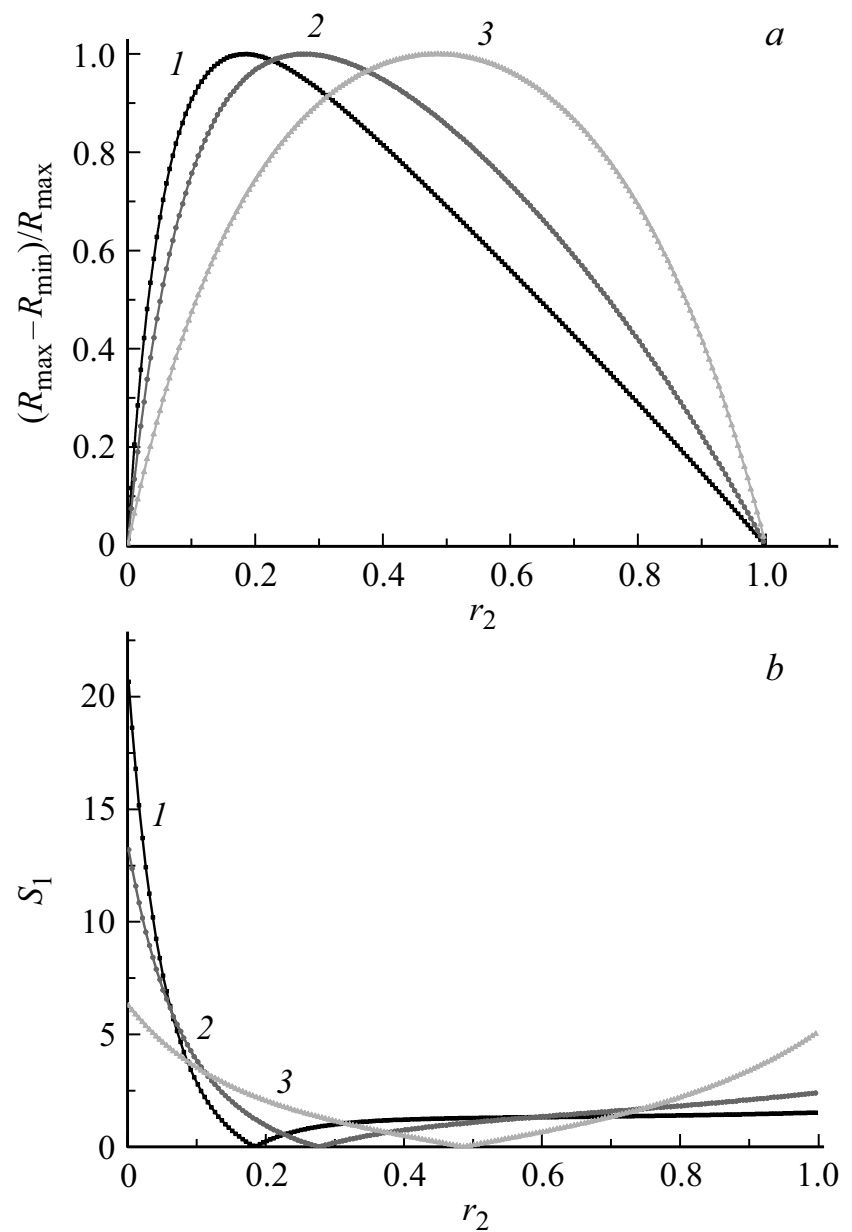

Рис. 2. Зависимость измеряемой величины (графики $a$ ) и чувствительности измерений $(b)$ от амплитуды коэффициента отражения $r_{2}$ для различных материалов переднего зеркала: 1 - кварц, 2 - лейкосапфир, 3 - селенид цинка. 
Чувствительность $S_{1}$ при измерениях $r_{2}$ определим величиной модуля производной:

$$
S_{1}=\left|d / d r_{2}\left(\Delta S / S_{\max }\right)\right|
$$

график которой как функции $r_{2}$ показан на рис. $2, b$ для тех же материалов переднего зеркала. Области высокой чувствительности измерений расположены вблизи малых $r_{2}$, а также вблизи $r_{2}$, близких к 1 . Для повышения чувствительности в узком диапазоне длин волн в качестве переднего зеркала можно использовать подложки с отражающими покрытиями, заранее определив их коэффициент отражения. Детектирующая способность определяется уровнем шума в измеряемом сигнале, т. е. ошибкой $\Delta r_{n}$ в измеряемом коэффициенте отражения $r_{2}$. Для обычных измерений $\Delta r_{n}=S_{0 n} / S_{0}$, где $S_{0}-$ сигнал от падающего пучка, а $S_{0 n}-$ шумовой сигнал. В тех же условиях для интерферометрических измерений

$$
\Delta r_{n}=\Delta r_{2}\left(\Delta S_{n} / \Delta S+S_{0 n} / S_{0}\right)
$$

т. е. ошибка $\Delta r_{n}$ пропорциональна малой величине $\Delta r_{2}$, что позволяетизмерять $r_{2}$ существенно точнее.

Необходимо отметить особенность регистрации отраженного от интерферометра сигнала. Падающий и отраженный от исследуемого образца пучки имеют в поперечном сечении гауссову форму без каких-либо флуктуаций, в то время как при отражении от интерферометра эти флуктуации появляются. Они обусловлены интерференцией из-за конечного размера пучка, т. е. дифракцией на интерферометре (в нашем случае размер пучка в области детектора составляет $\approx 2 \mathrm{~mm}$ ). Интерференция при отражении от интерферометра перераспределяет энергию падающего пучка по различным порядкам при сохранении полной энергии. В нашем случае на размере пучка наблюдается 5-6 порядков интерференции. При помещении перед фотодетектором диафрагмы диаметром $\leq 0.1 \mathrm{~mm}$ наблюдаются флуктуации $\Delta S / S_{\max }$ вблизи центра пучка $\sim 3 \%$, а на периферии пучка до 20\%. Таким образом, использование детекторов с размерами, меньшими размеров пучка, приведет к существенным ошибкам. В нашем случае вся энергия пучка попадает в размер $4 \mathrm{~mm}$. Используемый нами фотодетектор ФД-24К имеет размер светочувствительной площадки $1 \mathrm{~cm}$ при разбросе чувствительности по площади $\leq 3.9 \%$.

\section{Обсуждение результатов измерения}

В таблице показаны результаты измерений амплитуды коэффициента отражения $r_{2}$ по обычной и по интерференционной методикам. Там, где возможно, приведены результаты расчета исходя из справочных данных.

Измерения с просветленной пластиной дают завышенную величину коэффициента отражения при прямых измерениях по сравнению с интерференционными; это может свидетельствовать о заметном вкладе рассеяния от пластины, которое, естественно, не учитывается при интерференционном измерении.

В случае кварцевой пластины при интерференционном измерении мы попадаем в область максимума кривой на рис. 2, что приводит к большой ошибке и практически к неопределенности амплитуды коэффициента отражения. Результат прямых измерений несколько ниже расчетного, что может быть обусловлено влиянием бокового рассеяния, приводящего к потере сигнала, регистрируемого приемником.

Для зеркала с алюминиевым покрытием имеем близкие результаты. Для диэлектрического зеркала расхождение обусловлено неточностью прямых измерений двух близких по значению величин.

Для измерений, выполненных с полупроводниковыми образцами, в данном случае существенно, что энергия лазерного фотона выше энергии края поглощения. Фокусировка пучка на интерферометр приводит к повышению концентрации электронов в зоне проводимости до $10^{20} \mathrm{~cm}^{-3}$ для приграничной области, что, в свою очередь, приводит к существенным изменениям оптических характеристик, в том числе и коэффициента отражения. Это в меньшей степени сказывается на результатах прямых измерений, выполненных без фокусировки пучка.

B гетероструктуре на подложке GaAs некоторую ее часть занимают слои AlAs, имеющие более низкий показатель преломления, в которых не происходит межзон-

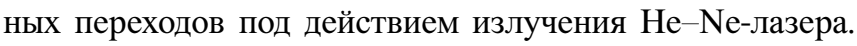
В прямых измерениях наблюдается даже снижение коэффициента отражения по сравнению с подложкой GaAs. При фокусировке излучения в интерферометр, вероятнее всего, проявляется нелинейность, аналогично образцу GaAs приводящая к росту коэффициента отражения. Строго говоря, сравнивать измерения прямые и те, что выполнены с помощью RI, нельзя, так как они проведены в разных условиях по интенсивности пучков.

Аналогичные измерения можно проводить и при наклонном падении пучка на образец, выбирая соответствующую поляризацию излучения. В этом случае при фокусировке на интерферометр основной проблемой для многолучевой интерференции будет уменьшение совпадения пучков, участвующих в интерференции. Поэтому имеет смысл перейти к двухлучевой интерференции, добиваясь полного несовпадения вторичных пучков. Для $\Delta S / S_{\max }$ получаются зависимости, аналогичные приведенным на рис. 2, $a$; методика измерений также сохраняется.

Оценим потенциальный выигрыш от использования предлагаемой методики на основе отражательного интерферометра на примере обнаружения слабой линии поглощения по спектру коэффициента отражения образца. Рассмотрим гетероструктуру на подложке арсенида галлия со слабой экситонной линией на длине волны $1.04 \mu \mathrm{m}$ (в области прозрачности GaAs). Оптические характеристики гетероструктуры зададим формулами 
Сравнение коэффициентов отражения, измеренных обычным способом (прямые измерения) и с помощью отражательного интерферометра

\begin{tabular}{|c|c|c|c|c|}
\hline \multirow{2}{*}{$\begin{array}{l}\text { Номер } \\
\text { образца }\end{array}$} & \multirow[b]{2}{*}{ Наименование образца } & \multicolumn{3}{|c|}{ Амплитуда коэффициента отражения $\left(r_{2}\right)$} \\
\hline & & расчет & $\begin{array}{c}\text { прямые } \\
\text { измерения }\end{array}$ & $\begin{array}{c}\text { интерференционные } \\
\text { измерения }\end{array}$ \\
\hline 1 & Просветленная пластина & - & 0.01243 & 0.00763 \\
\hline 2 & Кварцевая пластина & 0.1859 & 0.1784 & $\begin{array}{l}0.1238 \\
0.2583\end{array}$ \\
\hline 3 & Зеркало с алюминиевым покрытием & 0.9558 & 0.951 & 0.9461 \\
\hline 4 & Диэлектрическое зеркало & - & $>0.9785$ & 0.9981 \\
\hline 5 & Подложка GaAs & 0.5888 & 0.6085 & 0.6374 \\
\hline 6 & Гетероструктура на подложке GaAs & - & 0.5761 & 0.6331 \\
\hline
\end{tabular}

для действительной $n$ и мнимой $\kappa$ частей индекса преломления $n+i \kappa$ :

$$
\begin{gathered}
n=n_{0}+\left[A\left(\omega-\omega_{0}\right)\right] /\left[\left(\omega-\omega_{0}\right)^{2}+\Gamma^{2}\right], \\
\kappa=A \Gamma /\left[\left(\omega-\omega_{0}\right)^{2}+\Gamma^{2}\right] .
\end{gathered}
$$

Численные параметры близки к определяемым экспериментально значениям $n_{0}=3.492, A=1.22 \cdot 10^{11} \mathrm{~s}^{-1}$, $\Gamma=1.74 \cdot 10^{13} \mathrm{~s}^{-1}$. Будем рассматривать случай наклонного падения при угле падения, близком к углу Брюстера для $\mathrm{GaAs}\left(\sim 74^{\circ}\right)$ для $p$-поляризованного излучения, так что отражение от поверхности GaAs практически отсутствует.

На рис. 3 светлой линией показан спектр сигнала отражения при обычном способе измерения $R$, ось ординат расположена справа, единицы измерения $10^{-5}$. Здесь же приведен спектр сигнала интерферометра $R_{\mathrm{RI}}=\Delta R$, для которого ось ординат находится слева, единицы измерения $10^{-2}$. Как видно, в спектре сигнала при обычном способе измерения коэффициента отражения наблюдается дисперсионный контур малой (порядка $10^{-5}$ )

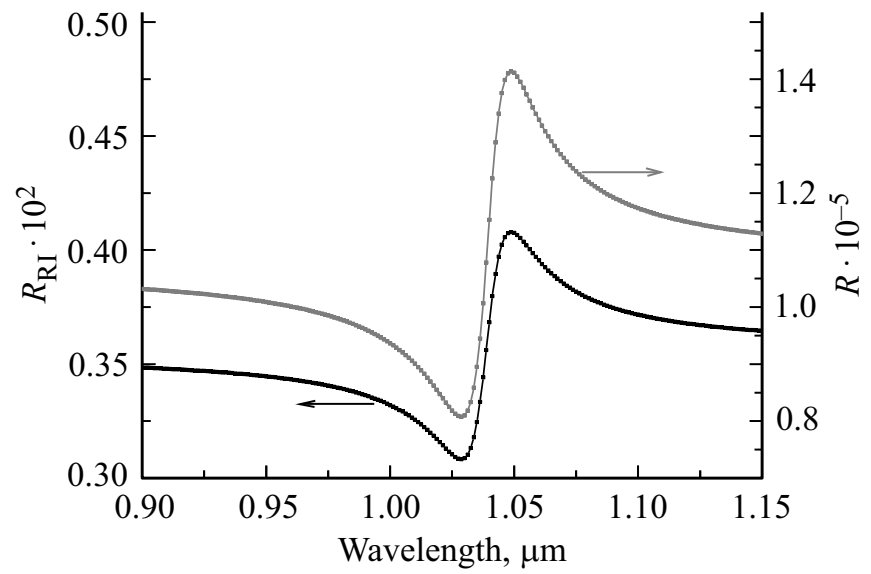

Pис. 3. Спектры отражения от поверхности образца, включающего слабую экситонную линию при измерении обычным способом $R$ (ось ординат справа, единицы измерения $10^{-5}$ ) и при измерении с помощью отражательного интерферометpa $R_{\mathrm{RI}}$ (ось ординат слева, единицы измерения $10^{-2}$ ). амплитуды. В спектре сигнала, получаемого с помощью отражательного интерферометра, форма линии та же, но амплитуда более чем в 100 раз выше.

\section{Заключение}

В работе подробно рассмотрен интерферометрический способ нахождения коэффициентов отражения оптических элементов с использованием отражательного интерферометра со сканируемой базой. Измеряемой величиной является $\left(S_{\max }-S_{\min }\right) / S_{\max }$, где $S_{\max }$ и $S_{\min }-$ значения максимумов и минимумов в сигнале отражения от сканируемого интерферометра Фабри-Перо. Проведены тестовые измерения для нескольких оптических элементов, в том числе для просветленной пластины, высокоотражающего зеркала и для полупроводниковых образцов. Проведен расчет ожидаемого интерферометрического сигнала от слабой линии поглощения в полупроводниковом образце, демонстрирующий преимущество предлагаемой методики по сравнению с обычным способом измерения коэффициента отражения.

\section{Список литературы}

[1] Борн М., Воль $\oint$ Э. Основы оптики. М.: Наука, 1970. 855 с.

[2] Троицкий Ю.В. Одночастотная генерация в газовых лазеpax. Новосибирск: Наука, 1975. 159 с.

[3] Троицкий Ю.В. // Квант. электрон. 1975. Т. 2. № 1. C. $2444-2452$

[4] Ковалёв А.А., Преображсенский В.В., Путято М.А., Пчеляков О.П., Рубцова Н.Н. // Изв. РАН, сер. физ. 2008. Т. 72. № 5. C. $771-773$. 